\title{
Focus on the Perforated Peptic Ulcer
}

\section{Sorin Cimpean*, Marie-Therese Marechal, Benjamin Cadiere, Gloire A Dieu Byabene, Luca Pau, Arianna Grilli, Guy-Bernard Cadiere}

Saint Pierre University Hospital, Brussels, Belgium

*Corresponding Author: Sorin Cimpean, Saint Pierre University Hospital, Brussels, Belgium.

Received: October 29, 2019; Published: November 11, 2019

DOI: 10.31080/ASGIS.2019.02.0098

\begin{abstract}
Peptic ulcer perforation is a life threatening complication of peptic ulcer disease. $10 \%-20 \%$ of patients with peptic ulcer will develop complications, only $2 \%-14 \%$ of the ulcers will perforate. The most common location for gastric perforation is the anterior wall (40\%) followed by the greater curvature (23\%), lesser curvature (15\%) and posterior wall (15\%). The abuse of NSAIDS and the infection with Helicobacter pylori are the two main reasons for the peptic perforation. The classic triad of sudden onset of abdominal pain, tachycardia and abdominal rigidity is the hallmark of PUD perforation. Abdominal CT scan with is considered as the gold standard for diagnosis of a perforation. Laparoscopic approach should be the first therapeutic option in patients with perforated peptic ulcer in a stable hemodynamic condition. The simple closure with or without omentoplasty and drainage is the most popular operation in case of simple perforation.
\end{abstract}

Keywords: Peptic Ulcer Disease; Helicobacter pylori; NSAIDS

\section{Introduction}

Peptic ulcer perforation is a life threatening complication of peptic ulcer disease (PUD). 10\%-20\% of patients with peptic ulcer will develop complications, only $2 \%-14 \%$ of the ulcers will perforate $[1,8]$. Perforation is one of the commonest causes of emergency hospitalization and surgery in peptic ulcer disease [1].

Perforated peptic ulcer is associated with short term mortality and morbidity in up to $30 \%$ and $50 \%$ of the patients. The incidence of perforated peptic ulcer is approximately 7-10 per 10.000 populations per year [2,3]. An estimated $2 \%$ to $10 \%$ of patients with PUD will present with perforation of the stomach or the duodenum in their lifetimes, with a disproportionately high risk for mortality in the elderly $[4,5]$. Acute perforations of the duodenum are estimated to occur in $2-10 \%$ of patients with PUD $[7,54]$.

The adjusted incidence rate for the overall 10-year period was 6.5 per 100000 per year and the adjusted mortality rate for the overall 10-year period was 1.1 per 100000 per year. However, for patient $\geq 60$ years, the incidence increased over 10 -fold, and mortality more than 50-fold, compared to younger ages [6,7]. Lifetime prevalence of perforation is about $5 \%$ with mortality ranging from $1.3 \%$ to $20 \%$ [8]. Mortality of perforation due to the secondary peritonitis ranges from 25\% in 1940 as reported by Bakey D., et al. Arveen S, Jagdish S., et al. (2009) in their study reported a global mortality of $8.5 \%$ [9].

\section{Material and Methods}

PubMed, Medline and Cochrane databases were researched from 2010 to 2019. Search items included peptic ulcer disease, peptic perforation, duodenal perforation, gastric perforation. Studies were included if they described the peptic perforation etiology, diagnostic, risk factors and treatment.

\section{Discussions}

The first recorded description of a peptic ulcer perforation was by the Princess Anne Henriette of England, the daughter of King Charles I of England and Princess Marie Henriette of France [10]. 
Peptic perforation is a serious complication of PUD. Patients with perforated peptic ulcer (PPU) often present with acute abdomen that carries high risk for morbidity and mortality [26]. Peptic perforations are a highly complex disease for which clinical prognoses are likely difficult. The incorporation of computerized learning systems might enhance clinical judgments to improve decision making and outcome prediction [11].

The most common location for gastric perforation is the anterior wall $(40 \%)$ followed by the greater curvature (23\%), lesser curvature (15\%) and posterior wall (15\%) [50]. For younger age, Hua., et al. found that of a total of 52 children, the site of perforation was the duodenum in 41 cases $(78.8 \%)$ and the stomach in the remaining 11 cases (21.2\%). Only 4 patients (7.7\%) had $H$. pylori infection. Wong., et al. found a strong male preponderance (88.2\%) among the 17 cases [12]. Svanes., et al. state that gastric ulcer perforations are associated with higher mortality than duodenal ulcer perforations. For Kumar., et al. PUD perforations greater than $5 \mathrm{~mm}$ are an independent risk factor for re-leak when a simple closure with omental patch alone is performed [13].

Perforated peptic ulcer can mimic acute pancreatitis, cholecystitis, or appendicitis when gastroduodenal contents track down the right paracolic gutter causing pain in the right iliac fossa. Perforated peptic ulcer is not initially considered in the differential diagnosis in up to $20 \%$ of proven cases [14].

Initially, the perforation leads to chemical peritonitis, with or without contamination with micro-organisms. Spillage of gastroduodenal contents is usually diffuse but may be localized in the upper abdomen. The intermediate stage appears after 6 to 12 hours and many patients obtain some spontaneous relief of the pain. This is probably due to the dilution of the irritating gastroduodenal contents by the ensuing peritoneal exudate. The intra-abdominal infection appears after 12 to 24 hours [30].

The overall mortality due to perforation peritonitis ranges between 6 and 27\%. One of the most important factors responsible for mortality is septicemia [15].

\section{Signs and symptoms}

The classic triad of sudden onset of abdominal pain, tachycardia and abdominal rigidity is the hallmark of PUD perforation [8].
The patient can present signs and symptoms of septic shock, such as tachycardia, hypotension, and anuria. The general signs of infection that can be found are shortness of breath, fever, chills or sweating, confusion.

The abdominal exam can find epigastric, right flank or generalized tenderness, rebound tenderness, guarding, and rigidity of the abdominal wall. The localization, the size of the perforation and the amount of bacterial and gastric or duodenal contents that contaminate the abdominal cavity can influence the general and abdominal findings. In case of old, decompensated peritonitis the abdominal rigidity can disappear. If the ulcer is small and is spontaneously seal, the patient can present just an epigastric of right flank pain, with no signs of infection. Symptoms such as nausea, vomiting, loss of appetite, radiating pain resulted from a wide range of mucous damage and stimulation of the nerves distributed throughout the peritoneum and mesentery [16]. Even slight movements can worsen the abdominal pain, and these patients prefer to rest in a fetal position.

\section{Diagnostic}

Abdominal X-ray exam, with the identification of free air under the diaphragm, has been the imaging procedure of choice historically with sensibility from $30 \%$ to $85 \%$ [17]. Conventional abdominal X-ray may not detect pneumoperitoneum or retroperitoneum in up to $49 \%$ of patients [18]. As little as $1 \mathrm{ml}$ of free intraperitoneal air can be detected on the erect radiography.

Ultrasonography can locate the site of ulcer perforation and the presence of free peritoneal fluid and air but the role in this type of pathology is limited. Still, the abdominal ultrasound has been found to be more sensitive to plain radiographs in the diagnosis of free intraperitoneal air [19].

Abdominal CT scan with contrast injection is considered the gold standard for the diagnosis of a perforation. Abdominal CTscan, have a sensibility between $70 \%$ and $98 \%$ and is important in differential diagnoses. The most relevant sign on CT scan are the "ligamentum teres sign" (free gas outlining the intrahepatic fissure and ligamentum teres, sign of perforation of the duodenal bulb or stomach), the "peri-portal free gas sign" (sign of upper gastro-intestinal tract perforation) and the "falciform ligament sign" (free gas or a gas-fluid level crossing the mid-line and accentuating the 
falciform ligament, sign of proximal gastro-intestinal tract perforation) $[18,19]$.

\section{Risk factors}

The precipitating factors are Helicobacter pylori infection, use of steroids, anti-inflammatory drugs, smoking, heavy and chronic alcoholic intake, trauma and gastric malignancies [20].

A prospective cohort study from Denmark found that smoking more than 15 cigarettes daily increased the risk of peptic ulcer perforation 3.5 times. A retrospective study from Norway reports that a cohort pattern in prevalence of smoking partly explained the cohort pattern in perforation risk for both sexes [10].

Non-Steroidal Anti-Inflammatory Drugs (NSAIDS) penetrate the gastric mucous membrane by inhibiting cyclooxygenase-1 (COX-1) and inhibit the production of prostaglandins in the stomach that play a critical role in the mucosal defenses of the stomach against acid and pepsin induced injury. Prostaglandins stimulate mucus and bicarbonate production, and regulate the gastric mucosal blood flow [21]. Use of steroids and older age are well known risk factors for mortality in perforated gastroduodenal ulcer whereas they were associated with a reduced risk of re-intervention. There is a protective effect of older age and steroid use and it might be related to the fact that elderly patients and those treated with steroids may display atypical clinical symptoms, which challenges the diagnosis of surgical complications [22]. Due to the high fetal mortality during the pregnancy in case of peritonitis due to an ulcer perforation, prophylactic steroid administration during the pregnancy as for significant leucopenia should be limited [23].

Chronic use of synthetic cannabinoids (SCs) which has become an increasingly prevalent problem can cause gastric and duodenal ulcer because of their effects on gastric secretion and emptying [24].

Patients with predominantly antral infection of $H$. pylori have negative feedback of acid secretion, resulting in increased gastric acid production with the apparition of duodenal and pre-pyloric ulcers. Patients with uniform infection have a low acid production secondary to inflammation of the gastric body which impairs the normal function of the acid secreting mucosal and the best conditions for gastric ulcers [21].
The abuse of NSAIDS and the infection with Helicobacter pylori are the two main reasons for PUD.

\section{Morbidity predictive factors}

Preoperatively the combination of age, active cancer, hyperbilirubinemia, hypoalbuminemia, elevated creatinine and delay from perforation to surgery of $>24 \mathrm{~h}$ are predictive factors of morbidity and mortality. Hypoalbuminemia is the strongest single predictor of mortality and may be included for improved risk estimation [25].

Despite their often severe medical co-morbidities, obese surgical patients and obese, critically ill patients have a lower mortality than normal-weight patients. This can be explained by the "obesity paradox". These patients have an insufficient nutritional reserve, but the chronic inflammatory provided by obesity can results in a more efficient metabolic state where the body is ready for the appropriate inflammatory response [26].

Concerning the postoperative morbidity there are multiples factors that influence the outcome a high ASA score, the presence of preoperative shock, an open surgery and a long operating time more than 150 minutes are high risk factors for morbidity. For the instable patients or who presents comorbidities, a damage control surgery with limited operative time is suitable [27].

Patients above the age of 60 , with a time to presentation longer than 24 hours, presence of shock at the time of presentation and concomitant diseases, and a perforation diameter wider than 0.5 $\mathrm{cm}$ are patients at high risk for post-operative morbidity and mortality [28]. Older patient age and comorbidities are the main factors leading to sepsis or death. A majority of factors can be assessed preoperatively. Thus, patient evaluation can be preoperatively performed using the duration of symptoms and CRP level in conjunction with patient age and comorbidity [29].

\section{Unusual presentations}

A very rare and severe presentation is the perforation of a peptic ulcer in a hiatal hernia with penetration into the pericardium. More than $50 \%$ of patients with penetrating gastric ulcer into the pericardium die secondary to uncontrolled massive internal hemorrhage, septic peritonitis, or prolonged multisystem organ failure due to hypovolemic or cardiogenic shock [29]. 
There are also described subclinical forms of PUD perforation and the patient can present directly a subphrenic abscess [30].

In case of Roux-en-Y Gastric Bypass (RYGB) as the excluded stomach is challenging to reach, aggravating factors for peptic perforation, such as eradication of $H$. pylori must be treated before the surgery. In patients with recurrent, unexplained epigastric pain, the presence of and peptic ulcer or even perforation has to be taken into consideration. In case selected cases an exploratory laparoscopy or endoscopic evaluation of the gastric remnant via temporary gastrostomy (during diagnostic laparoscopy) must be taken in consideration [31].

Treatment

Farthmann., et al. described therapeutic principles in the surgical management of intra-abdominal infections: controlling contamination source, contamination diminution by the reduction or elimination the bacterial load and treatment of residual and prevention of recurrent infection with antibiotics [32,35].

In high-risk patients, those who would not tolerate or cannot undergo general anaesthesia, or for patients with a sealed retroperitoneal perforation, a non-operative approach ca be the best option. This treatment consists in proton pomp inhibitor (PPI) and antibiotic, a nasogastric tube and percutaneous drainage of any collections. For the elderly, the mortality rate is over 50 per cent for both operative and conservative approaches [33].

In view of preoperative and intra-operative diagnostic uncertainty, along with the lack of full staging of potential malignancy (including lymphoma) and the controversy as to the place of resection for cure; patch repair and biopsy is recommended where possible. Follow-up endoscopy should be routinely carried out in the majority of patients after discharge home to ensure healing and exclude malignancy [34].

Candida has been recovered in $29 \%-57 \%$ of peritoneal fluid cultures in patients with peptic perforation. Candida in peritoneal fluid cultures may be associated with worse outcomes in patients with perforated peptic ulcers (PPUs) [35].

The postoperative therapy must include PPI (proton pump inhibitor) and the treatment of Helicobacter pylori if positive.

\section{Surgical treatment}

Johan Mikulicz-Radecki (1850-1905) reported the closure of a peptic perforation by simple suture. In 1843, Edward Crisp was the first to report a series of 50 cases of PPU and accurately summarized the clinical aspects of perforation. Until the discovery of the role of H. pylori in gastric and peptic ulcers by Barry J. Marshall and Robin Warren in 1982, stress and life style factors were believed to be the most important factors [36].

Definitive surgical procedures, including antrectomy, have been routinely performed for decades in Japan, other Asian countries, and Eastern Europe; nowadays, non-definitive surgical procedure like simple closure with or without omentoplasty and drainage are the most popular in case of perforation [37].

Small perforations that are less than $1 \mathrm{~cm}$ in size, and have the best outcome; large perforations have a size between $1 \mathrm{~cm}$ and 3 $\mathrm{cm}$ and giant perforations that exceed $3 \mathrm{~cm}$ size. Generally, the surgical options are suture of the perforation with omentoplasty, resection of the perforation in the form of a partial gastrectomy, with reconstruction as either a Billroth I or II anastomosis. In severe case more aggressive and morbid procedures are gastric disconnection, antrectomy, gastrostomy, lateral duodenostomy and feeding jejunostomy with restoration of intestinal continuity electively [38] The decision to use pyloric exclusion to repair a duodenal injury is limited in cases of severely injured patients, with effective exclusion of the duodenum until after healing has occurred. Certain factors may lead surgeons to consider an injury severe and order a complex procedure, including blunt trauma or bullet wounds, delay to repairs exceeding 24 hours, injury of the first or second portions of the duodenum, duodenal injuries associated injuries to the pancreas or common bile duct (or both) and compromised blood supply to the duodenum [39].

In case of peptic ulcer disease perforations larger than $2 \mathrm{~cm}$ have been shown to have higher leak rates (up to 15\%) with primary repair, with an associated mortality of $10-35 \%$ increasing with delay in re-exploration [40].

Omental patching does not correct the underlying disease process which causes foregut perforation, and has a $12 \%$ endoscopically proven recurrent ulceration rate and a $23 \%$ incidence of recurrent symptoms within 44 months [41]. 
Using a drain won't avoid the need for reoperation if leakage develops, but it may provide early warning if there is a leak that requires reoperation. The sub hepatic drain cannot prevent leakage from the duodenal suture line; it can control the source of infection by creating a controlled external fistula. If patient presents generalized intra-abdominal collection, we usually prefer a subhepatic and an additional pelvic drain [42].

For the gastric ulcer perforation, a biopsy from the ulcer margin is recommended to exclude the risk of underlying malignant process.

\section{The place of laparoscopy}

Laparoscopic approach should be the first therapeutic option in patients with perforated peptic ulcer after considering other variables such as experience, costs and availability [45]. The laparoscopic approach allows better visualization, is less traumatic and allows quick recovery after the operation. Wong., et al. published a case series highlighting two of the main advantages of laparoscopic approach: first, the advantage of pathology identification when there is uncertainty of the diagnosis, along with the avoidance of misplacing an abdominal incision [46].

Negative factors for the laparoscopic approach are shock, delayed presentation ( $>24 \mathrm{~h}$ ), confounding medical conditions, age > 70 years, ASA (American Society of Anesthesiologists) score III-IV and Boey score of $\geq 2$, ulcer location or large perforation size ( $>$ 6-10 mm). The most accepted contraindication is signs of shock due to the negative impact of increase duration of surgery and negative influence of pneumoperitoneum on renal function [47]. For the laparoscopic approach the concern is the pneumoperitoneum for $\mathrm{CO}_{2}$ who leads to increased intra-abdominal pressure intraoperatively and is related to increased risk of bacteremia and sepsis due to the increased chance of bacterial translocation of peritoneal cavity into the bloodstream, increasing the occurrence of pneumonia in patients submitted to this approach [48].

In laparoscopy the most common cause of conversion was an inability to repair the ulcer due to either technical difficulties or size of perforation. The conversion rates are directly influenced by the laparoscopic skills and experience of the surgeon. The conversion rate range is described between 2.6 to $7.7 \%$ [49].
Because of the learning period, laparoscopic surgery may not be suited to every surgeon. When performed by laparoscopic experts, can be a valuable approach for treating difficult duodenal ulcer perforations [50,51]. Bertleff and Lange suggest that a number of 20-25 cases for the surgeon operating with the laparoscopic method are sufficient to achieve an acceptable level of expertise [52,53].

The laparoscopic approach is without any doubt an effective method for treatment of perforated duodenal ulcer in selected cases [54].

\section{Endoscopy}

The hybrid endoscopic and surgical procedures with high rates of luminal perforations stimulate the development of more effective and user-friendly devices for endoscopic closure of perforations [43].

Endoscopic treatment for small gastric defects $(<10 \mathrm{~mm})$ using TTS (through-the-scope clips) with a linear shape, is an acceptable method and should be attempted. Endoscopic treatment for large gastric defects (>10mm) by the OTSC (over-the-scope clip system) can be efficient in duodenal perforation [44].

\section{Conclusion}

Perforated peptic ulcer is a severe condition with high morbidity. Early presentation of the patient to the hospital and early surgical therapy is very important for a good outcome. Abdominal CT scan with is considered as the gold standard for the diagnostic of perforation. Exploratory laparoscopy with suture and omentoplasty is the most popular technique in case of simple perforation to a hemodynamic stable patient.

\section{Bibliography}

1. Dongo AE., et al. "A Five-Year Review of Perforated Peptic Ulcer Disease in Irrua, Nigeria". International Scholarly Research Notices (2017): 1-6.

2. Muller MK., et al. "Perforated Peptic Ulcer Repair: Factors Predicting Conversion in Laparoscopy and Postoperative Septic Complications". World Journal of Surgery 40.9 (2016): 21862193.

3. Bertleff MJOE and Lange JF. "Perforated Peptic Ulcer Disease: A Review of History and Treatment". Digestive Surgery 27.3 (2010): 161-169.

4. Chung KT and Shelat VG. "Perforated peptic ulcer - an update". WJGS 9 (2017): 1. 
5. Schulte-Hermes M., et al. "Acute tension pneumopericardium due to perforated gastric ulcer without diagnostic radiographic findings $72 \mathrm{~h}$ before perforation". Journal of Cardiology Cases 18 (2018): 201-203.

6. Lee CW and Sarosi GA. "Emergency Ulcer Surgery". Surgical Clinics of North America 91.5 (2011): 1001-1013.

7. Bhattacharjee HK., et al. "Duodenal perforation following blunt abdominal trauma". 5 (2011).

8. Thorsen K. "Epidemiology of perforated peptic ulcer: Age- and gender-adjusted analysis of incidence and mortality". WJG 19 (2013): 347.

9. Byrge N., et al. "Laparoscopic versus open repair of perforated gastroduodenal ulcer: a National Surgical Quality Improvement Program analysis". The American Journal of Surgery 206 (2013): 957-963.

10. Grišin E., et al. "Laparoscopic pyloroplasty for perforated peptic ulcer”. Wiitm 3 (2017): 311-314.

11. Søreide K., et al. "Perforated peptic ulcer". Lancet 386 (2015): 1288-1298.

12. Quah GS., et al. "Laparoscopic Repair for Perforated Peptic Ulcer Disease Has Better Outcomes Than Open Repair". Journal of Gastrointestinal Surgery 23 (2019): 618-625.

13. Ersoz N and Kilbas Z. "To drain or not to drain in perforated peptic ulcer". Journal of Experimental and Integrative Medicine 2.1 (2012): 45.

14. Bali RS., et al. "Perforation Peritonitis and the Developing World”. ISRN Surgery (2014): 1-4.

15. Gupta S., et al. "The management of large perforations of duodenal ulcers". BMC Surgery 5 (2005): 15.

16. Tanaka R., et al. "Treatment for Perforated Gastric Ulcer: a Multi-institutional Retrospective Review". Journal of Gastrointestinal Surgery 17 (2013): 2074-2081.

17. Thorsen K., et al. "What Is the Best Predictor of Mortality in Perforated Peptic Ulcer Disease? A Population-Based, Multivariable Regression Analysis Including Three Clinical Scoring Systems". Journal of Gastrointestinal Surgery 18 (2014): 12611268.

18. Smith D., et al. "The natural history of perforated foregut ulcers after repair by omental patching or primary closure". European Journal of Trauma and Emergency Surgery 44 (2018): 273-277.
19. Aboobakar MR., et al. "Gastric perforation following blunt abdominal trauma”. Trauma Case Reports 10 (2017): 12-15.

20. Duch P and Møller MH. "Body mass index is not associated with reoperation rates in patients with a surgically treated perforated peptic ulcer". 5 (2015).

21. Fraga GP., et al. "The use of pyloric exclusion for treating duodenal trauma: case series". Sao Paulo Medical Journal 126 (2008): 337-341.

22. Rohit DK., et al. "Clinical study and management of peritonitis secondary to perforated peptic ulcer". International Journal of Surgery 4 (2017): 2721.

23. Rani J. "Perforated Duodenal Ulcer - A Rare Cause of Acute Abdomen in Pregnancy". JCDR (2014).

24. Schein M. "Perforated Peptic Ulcer". In: Schein M, editor. Schein's Common Sense Emergency Abdominal Surgery: A Small Book for Residents, Thinking Surgeons and Even Students. Berlin, Heidelberg: Springer Berlin Heidelberg (2000): 97-105.

25. Kuzmich S., et al. "Perforated Pyloroduodenal Peptic Ulcer and Sonography”. American Journal of Roentgenology 199 (2012): W587-594.

26. Ansari D., et al. "Diagnosis and management of duodenal perforations: a narrative review". Scandinavian Journal of Gastroenterology 54 (2019): 939-944.

27. Unver M., et al. "Prognostic Factors in Peptic Ulcer Perforations: A Retrospective 14-Year Study". International Surgery 100 (2015): 942-948.

28. Coppolino F., et al. "Gastrointestinal perforation: ultrasonographic diagnosis". Critical Ultrasound Journal 5 (2013): S4.

29. Schwartz S., et al. "Perforated Peptic Ulcer in an Adolescent Girl”. Pediatric Emergency Care 28 (2013): 709-711.

30. Kutlu OC., et al. "The successful use of simple tube duodenostomy in large duodenal perforations from varied etiologies". International Journal of Surgery Case Reports 4.3 (2013): 279282.

31. Irene M., et al. "Intra- abdominal sepsis from a perforated duodenal ulcer-Management of a difficult surgical abdomen”. International Journal of Surgery Case Reports 55 (2019): 171173. 
32. Stepanyan SA., et al. "Laparoscopic and open repair for perforated duodenal ulcer: single-center experience". Wiitm 14 (2019): 60-69.

33. Yamamoto K., et al. "Evaluation of risk factors for perforated peptic ulcer". BMC Gastroenterology 18 (2018): 28.

34. Dodiyi-Manuel A., et al. "Presentation and management of perforated peptic ulcer disease in a tertiary centre in South Nigeria". Journal of the West African College of Surgeons 5 (2015): 36-48.

35. Kim MG. "Laparoscopic Surgery for Perforated Duodenal Ulcer Disease: Analysis of 70 Consecutive Cases from a Single Surgeon". Surgical Laparoscopy Endoscopy and Percutaneous Techniques 25 (2015): 6.

36. Munoz Abraham AS., et al. "Incidence and Outcomes of Perforated Peptic Ulcers in Children: Analysis of the Kid's Inpatient Database and Report of Two Cases Treated by Laparoscopic Omental Patch Repair". Journal of Laparoendoscopic and Advanced Surgical Techniques 29 (2019): 248-255.

37. Gurusamy KS and Pallari E. "Medical versus surgical treatment for refractory or recurrent peptic ulcer". Cochrane Database of Systematic Reviews (2016).

38. Sanabria A., et al. "Laparoscopic repair for perforated peptic ulcer disease". 21 (2010).

39. Leeman MF., et al. "The management of perforated gastric ulcers". International Journal of Surgery 11 (2013): 322-324.

40. Di Saverio S., et al. "Diagnosis and treatment of perforated or bleeding peptic ulcers: 2013 WSES position paper". World Journal of Emergency Surgery 9 (2014): 45.

41. Søreide K., et al. "Predicting outcomes in patients with perforated gastroduodenal ulcers: artificial neural network modelling indicates a highly complex disease". European Journal of Trauma Emergency Surgery 41 (2015): 91-98.

42. Buyukbese Sarsu S. "Unusual side effect of cannabis use: acute abdomen due to duodenal perforation". International Journal of Emergency Medicine 9 (2016): 18.

43. Horn CB., et al. "Pre-Operative Anti-Fungal Therapy Does Not Improve Outcomes in Perforated Peptic Ulcers". Surgical Infections 19 (2018): 587-592.
44. Deshmukh S., et al. "Study of the effect of post-operative medical management on peptic ulcer in patients of perforated peptic ulcer disease". International Surgery Journal (2016): 12671272.

45. Sweeney KJ., et al. "Surgical management of perforated peptic ulcer disease". Irish Journal of Medical Science 175 (2006): 5054.

46. Hasselager RB., et al. "Risk factors for reintervention after surgery for perforated gastroduodenal ulcer: Reintervention after surgery for perforated gastroduodenal ulcer". British Journal of Surgery 103 (2016): 1676-1682.

47. Camera L., et al. "Perforated duodenal ulcer presenting with a subphrenic abscess revealed by plain abdominal X-ray films and confirmed by multi-detector computed tomography: a case report". Journal of Medical Case Reports 7 (2013): 257.

48. Pohl D., et al. "Perforated duodenal ulcers after Roux-Y Gastric Bypass". The American Journal of Emergency Medicine 36 (2018): 1525.e1-1525.e3.

49. Tan S., et al. "Laparoscopic versus open repair for perforated peptic ulcer: A meta analysis of randomized controlled trials". International Journal of Surgery 33 (2016): 124-132.

50. Søreide K., et al. "Strategies to improve the outcome of emergency surgery for perforated peptic ulcer: Emergency surgery for perforated peptic ulcer". British Journal of Surgery 101 (2014): e51-64.

51. Tas I., et al. "Risk factors influencing morbidity and mortality in perforated peptic ulcer disease". UCD 31.1 (2015): 20-25.

52. Paspatis G., et al. "Diagnosis and management of iatrogenic endoscopic perforations: European Society of Gastrointestinal Endoscopy (ESGE) Position Statement". Endoscopy 46 (2014): 693-711.

53. Parsi MA., et al. "Closure of a perforated duodenal ulcer by over-the-scope clip". Gastrointestinal Endoscopy 81.4 (2015): 1020-1021.

54. Milosavljevic., et al. "Complications of Peptic Ulcer Disease". Digestive Disease 29.5 (2011): 491-493.

\section{Volume 2 Issue 10 December 2019 (C) All rights are reserved by Sorin Cimpean., et al.}

